Universidad de Costa Rica
Facultad de Educación
Instituto de Investigación para el Mejoramiento
de la Educación Costarricense

\title{
LOS “MACHETES” EN LA TERCERA REVOLUCIÓN. REPENSANDO LA EDUCACIÓN
}

\section{Rolando Quesada Sancho'}

Desde hace algunos años, se viene poniendo en el "banquillo de los acusados" a la Educación, aduciendo, entre otras cosas, que carece de calidad y que por eso la sociedad ha entrado en crisis; así la responsabilizan de un cúmulo de decisiones y desfaces producidos en el mundo del trabajo y otros espacios. Las acusaciones tienden a analizar el "fenómeno educativo", desde una perspectiva descontextualizada, como un hecho aislado, elaborando con ello una abstracción de esa parte de la totalidad a la cual pertenece.

Los análisis elaborados de esa forma, podrían establecer lógicas aparenciales que justifican esa culpa. Pero la problemática no pasa solo por la calidad de la educación, sino que está atravesada por una cadena de circuitos que pueden hacer de la educación un factor de desarrollo económico, social y cultural o un factor retardatario y poco coherente con las realidades nacionales.

Desde este escenario, entonces, es lícito preguntarse acerca de las transformaciones en la estructura productiva que están sucediendo en nuestras realidades, con el fin de perfilar de una mejor manera la cualificación que se debe ofrecer a los y las ciudadanas en correspondencia con las necesidades de nuestra sociedad y las demandas del mercado. Es decir, se trata de identificar la correlación entre los saberes que se ofrecen desde la escuela y las nuevas necesidades y demandas.

\footnotetext{
${ }^{1}$ Antropólogo-Universidad de Costa Rica. Máster en Ciencias Sociales con énfasis en Educación, Flacso-Argentina. Investigador del Centro de Evaluación Académica y del Instituto de Investigaciones para el Mejoramiento de la Educación Costarricense. Profesor de la Escuela de Antropología y Sociología.
} 
Sospechamos que estas transformaciones se reflejan en los cambios en el mundo del trabajo, de tal suerte que ambas (necesidades y demandas) no sobreviven independientemente. Esto, aunado a los lineamientos de los organismos multilaterales, son aspectos en los que debemos reflexionar toda vez que abordemos el tema de educación, para decidir acerca de los contenidos o saberes que respondan a las solicitudes sociales.

Desde este momento podemos asegurar que ponemos pie en una arena polémica, en tanto que:

- Si educamos solo para el trabajo, pareciera que adherimos a los supuestos de los Organismos Multilaterales.

- Si educamos para la vida, corremos el riesgo de que las competencias adquiridas por las personas no coincidan con las solicitadas por el mundo del trabajo.

- Si seguimos con el sistema vigente, pareciera que la subocupación o subempleo es inminente y ascendente, con el riesgo de formar para la frustración.

- Si adherimos a la resistencia, peligrosamente nos podríamos acercar a la exclusión, en tanto que los capitales extranjeros que aterrizan en nuestro suelo solicitan otro tipo de trabajador.

Lo que si estamos seguros es que todos los puntos anteriores convergen en la necesidad y derecho que todos y todas las ciudadanas tenemos: el trabajo. Este derecho reinvindica la base fundamental del ser ciudadano, pues estar desempleado o desempleada significa ser una persona excluída, no solo del mundo del trabajo sino de otros espacios como el de la salud, la vivienda, el jurídico, la recreación, la jubilación, entre otros, y del civil solo nos quedaría el derecho al voto, que por otro lado es el único punto de convergencia de ciudadanía que tendríamos las personas excluídas e incluídas, probablemente porque existen todavía intereses políticos de que así sea.

Trabajaremos aquí la premisa de que, en tanto ser humano ciudadano, se tiene el derecho a un trabajo, a permanecer incluído y la educación, en tanto institución pública, 
debe contribuir en la formación de los hombres y las mujeres para que puedan acceder a un trabajo digno y ejercer la ciudadanía.

\section{1. ¿Se estarán diluyendo los machetes?}

Un punto de partida para dilucidar la relación existente entre las transformaciones científicas y la cualificación de la mano de obra, remite al análisis de las condiciones de la educación antes de las últimas transformaciones científico-tecnológicas mundiales, que dinamizan los procesos productivos.

De acuerdo con Tedesco (1995) la escuela se proponía brindar informaciones, conocimientos, valores, actitudes, habilidades, etc. cada vez más especializados para que las personas pudieran desempeñar diversos roles sociales los cuales se hallaban en una estructura ordenada jerárquicamente. La formación que recibía la persona respondía tanto a las necesidades sociales como a las demandas del mercado, su formación le permitía desempeñarse en un reducido rango de acción laboral, que dada las condiciones del mundo del trabajo, no era indispensable apropiarse de recursos para optar por la movilidad hacia otros sectores.

Esto daba una doble seguridad, por un lado la instancia empleadora se garantizaba que el trabajador pasaría un período prudencial en el puesto para el cual era capacitado y por otro lado el trabajador se apropiaba del puesto por razones varias, entre ellas por su cualificación.

Esas competencias que ofrecía la escuela muestran signos de caer en obsolescencia, dada la suerte que siguen el modelo fordista y taylorista. Nos encontramos pues ante un nuevo conjunto de innovaciones, cruzando el umbral de una nueva puerta que probablemente nos lleve a situaciones complejas -por ahora- y hasta con simulaciones dolorosas. 
¿En qué consiste este conjunto de novedades? Aspiazu (1988) expone la Tercera Revolución Industrial como un nuevo paradigma que permite aumentar la productividad con costos reducidos; el factor llave debe entonces, buscar un costo relativamente bajo con tendencia decreciente; una oferta aparentemente ilimitada en términos prácticos; universalidad de uso, masiva y evidente, bajo costo de insumos y mano de obra y ser factor base de un conjunto de innovaciones tecnológicas. Según el autor, el factor que cumpliría tales características y que además está relacionado con la acumulación de capital es el complejo electrónico.

Las tendencias centrales de este paradigma son: la concentración del poder en las grandes organizaciones, la creciente distribución asimétrica del ingreso entre clases sociales y naciones, y la reducción o anulación de la autonomía de aquellas naciones, precisamente donde las mayores organizaciones no tienen su núcleo principal de dirección, actividad y desarrollo (Op.Cit.; 28).

Este paradigma perteneciente a las grandes organizaciones, se afinca en cada uno de los países pobres, en un ritmo e intensidad coherente con la especificidad de sus propias realidades, por lo que la instalación de las nuevas transformaciones científico-tecnológicas no se producen "de un día para otro", sino que es un proceso paulatino que obliga a los países a realizar adaptaciones tanto económicas, como culturales, sociales, políticas, entre otros. Para lo cual se necesita que se reconfigure el modelo productivo vigente y se abran portillos o "flexibilicen" las estructuras y así permitir el ingreso de este nuevo extranjero: "el complejo electrónico".

Este nuevo paradigma productivo supone la utilización y la producción de ciencia y tecnología; sin embargo dada las condiciones económicas de los países como Costa Rica, la experiencia refleja que la utilización de una tecnología sofisticada es resorte de las filiales de las grandes empresas globales, y por tanto importadas, más que invención o desarrollo científico local. Esto nos hace suponer que nos encontramos con una realidad compleja, 
por un lado pretendemos utilizar tecnología de punta, pero por otro lado no poseemos capacidad objetiva para producirla y en la mayoría de los casos, para que sea utilizada por organizaciones productivas propias, lo que nos lleva a pensar que la lógica que imponen a los países pobres, es la de mantener el escenario (estabilidad política, sistema democrático, mano de obra cualificada, etc) para que "aterricen" los capitales extranjeros, y los países pobres aceptar esas condiciones para no quedar excluidos de la globalización económica².

Con la instalación de esta lógica, se reduce la soberanía nacional, y se imponen nuevas u otras formas de mirar el mundo, resignificando de esta manera la vida cotidiana. Lo que podría llevar a pensar que se dibujan dos opciones extremas de existencia para los países pobres, una soberana y excluída otra invadida pero incluída. Esta antinomia subyace en lo que en este documento tratamos de abordar, sin embargo ponemos el acento, como ya se indicó en el derecho al trabajo y las nuevas transformaciones en el sector productivo.

Con la incorporación de la nueva tecnología, se crea la necesidad de remozar habilidades, destrezas y conocimientos -recualificarnos- que nos permitan operar en este nuevo modelo. Esta situación controversial, por supuesto, parece deslegitimar el modelo vigente que, si bien es dominante en este momento, vertiginosamente está cayendo en decadencia.

Nos acercamos entonces a un movimiento dual, por un lado se exigen nuevos conocimientos a los trabajadores pero por otro se los descualifica, estimando que los saberes acumulados entran en desuso. Esto nos lleva a pensar -interpretando a Paiva (1994)- que el capitalismo de organización, esas condiciones productivas que recién comenzamos a dejar atrás, no han permitido una cualificación permanente de la fuerza de

\footnotetext{
${ }^{2}$ En países como el nuestro, esta situación se agrava más donde los empresarios y el mercado en general consideran que los "costos sociales" son muy elevados y buscan salidas para evadirlas o para alejarse de nuestro país hacia países aún más pobres pero más fácilmente explotados.
} 
trabajo, lo que podría estar abonando al argumento de que la especialización produjo una suerte de parálisis educativa en el trabajador ${ }^{3}$.

Parece entonces que en este movimiento, en donde convive el capitalismo de organización con el nuevo paradigma, parte de la fuerza de trabajo cualificada para el primer modelo, logra permanecer en él, atendiendo aquellas instancias productivas que sobrevivirán en el nuevo paradigma con viejas estructuras, otra parte se recualificará para incorporarse al mundo de la electrónica, mientras otros quedarán excluidos de estos movimientos, por falta de capacitación para activar y manipular las complejas máquinas electrónicas que fueron creadas para realizar acciones simples que, ordinariamente las realizaban las personas, hoy en vías de ser desplazadas ${ }^{4}$.

En este interjuego teñido de incertidumbre, es probable que aquellos que dispongan de un rico reservorio material y simbólico -aquellas personas que han tenido acceso a una amplia educación con "calidad"- tengan mejores condiciones para incorporarse al nuevo modelo. No obstante parte de los excluidos tratarán de aunarse a ellos cualificándose y permaneciendo como ejército de reserva, que entrarán y saldrán del proceso productivo según el caudal de capitales que "aterricen" en los países y la cualificación necesaria. Esto como correlato del grado de inclusión de nuestros países en el nivel global. Esto supone también que los jóvenes que recién se egresan del sistema educativo, vayan desplazando hacia el ejército de reserva o excluyendo, a las generaciones más viejas que no han podido recualificarse. En este camino, queda dibujado el "efecto dominó".

D. Aspiazu (Op.Cit.; 22-23) sostiene que la tendencia de este proceso es de reducir mano de obra calificada, puesto que "en la mayoría de los casos, simultáneamente con la

\footnotetext{
${ }^{3}$ No obstante, la discusión aporta contrargumentos provenientes de otras tesis, que hacen notar la existencia de una cualificación relativa con la implementación del fordismo, toda vez que el trabajador tuvo que adaptarse a una rotación en un mismo circuito laboral. Sin embargo, insistimos, esa cualificación cae paulatinamente en obsolescencia.

${ }^{4}$ Este proceso no es ninguna novedad, por el contrario es de larga data, su existencia puede tener alrededor de treinta años, pero actualmente es más grave y violento.
} 
reducción de los costos salariales y el empleo, se produce una polarización de perfil de calificación de la fuerza de trabajo", donde la caída del empleo se acentúa en el tramo que incluye a los obreros y personal calificado y es menor en aquellas personas no calificadas y en las profesionales (en ingeniería, en física o en formaciones técnicas), la Tercera Revolución Industrial nos está empujando a repensar los contenidos de la cualificación actual y la relación con el mercado y la vida en general.

La convocatoria de la Tercera Revolución, en el caso de Costa Rica, se centra en buscar el tipo de cualificación, necesaria para estar incluidos en los procesos, que se experimentan en forma diferencial en cada una de las regiones o Estado-nación. Esta nueva cualificación va más allá de habilidades y conocimientos concretos, etc. como se anotó, que satisfaga las nuevas demandas del mercado. Es una opción que se oriente a que las personas entiendan, piensen y hablen de aquello que pueden hacer, para lo cual hay que evitar la escisión entre conocimiento y pensamiento (Tedesco; 1995).

Filmus asegura que "la escuela no está en condiciones de promover trabajo para todos. Pero sí puede generar condiciones para que todos sean "empleados"... Ella puede contribuir a generar las condiciones para que sea la capacidad, y no el origen socioeconómico del niño, la que determine sus posibilidades de acceso a un buen trabajo" (Clarín; 28-3-96). Esto puede interpretarse como que es la escuela una instancia que debe formar no solo para acceder a un puesto laboral, sino para que las personas puedan generar cambios en las condiciones de su propia existencia, reiterando la premisa de que la escuela, puede asegurar una aprehensión por parte del y de la ciudadana, de saberes no solo para desenvolverse en la vida en forma crítica sino que tenga acceso a un trabajo digno.

Entonces ¿Estar incluídos o estar excluídos?, esa es la pregunta; la respuesta estará en los machetes educativos que se ofrezcan y de los que la persona logre apropiarse.

\section{Molejón, esmeril, laser: ¿con qué se afilan los machetes?}


Se ha insinuado, en el apartado anterior, el supuesto de que, los países como Costa Rica se convertirán en campos de aterrizaje de capitales extranjeros. Esto porque la nueva reconfiguración económica y social mundial nos estruja hacia allí. Al ingresar en esa lógica de mercado planetario, en tanto país pobre, el precio que se paga para permanecer incluídos es muy alto, pues en cierta medida se trata de crear y mantener condiciones adecuadas y atractivas para atraer y retener esos capitales.

No obstante, las condiciones nuestras distan mucho de las solicitudes globales. Según el informe "Estado de la Nación en desarrollo humano sostenible" (1995) en nuestro país se está experimentando una movilidad de contingentes laborales hacia sectores de la industria y los servicios. Esta situación plantea nuevas necesidades, por lo que, según consideran los expertos, tanto la capacitación como la educación deben ofertar recursos para la competitividad basada en conocimientos especializados, es por eso que se ha invertido en laboratorios y en formación de personal especializado.

Simultáneamente, se reconocen también en el mismo documento, que la inversión en investigación y desarrollo es muy baja por lo que el grado de actualización tecnológica también es baja. Como particularidad anotan que hay voluntad gubernamental, de las universidades y los sectores productivos para aprovechar la rica diversidad biológica (en turismo).

Con la apertura del mercado, las empresas deben ofrecer mejor producto con tecnología apropiada, sin embargo éstas no tenían capacidad para lograr innovaciones y proveer bienes y servicios competitivos, terminando por importarlos. Esto es clara evidencia de que en el país no ha existido una "política industrial y tecnológica que defina qué sectores se desea incentivar, lo mismo, que las medidas concretas para lograrlo, en función de la inversión"(Op.Cit.; 1995:36) en tecnología e investigación.

Dos años más tarde se insiste en que "La apertura económica, característica de esta etapa del desarrollo costarricense, exige a los productores establecidos en territorio 
nacional niveles elevados de productividad. Esto, a su vez, requiere niveles crecientes de formación y capacitación del recurso humano, así como también mecanismos que generen nuevas oportunidades a la fuerza de trabajo que se incorpora por pirmera vez en el mercado laboral" (E.L.N.;1997, p. 111).

Se destaca la decisión de INTEL de instalar una planta de producción en el país, esto con base en las ventajas competitivas que tienen en materia educativa, telecomunicaciones, régimen de zonas francas. "Esta decisión es importante no sólo por la inversión y el empleo a que dará lugar en forma directa, sino porque se espera que atraiga a otras empresas internacionales de alta tecnología" (Op.Cit.; 1997, p. 37)

Existe una fuerte preocupación por generar empleos pues tal como se evidencia en el documento citado, "por tercer año consecutivo, -ha- aumentado el desempleo abierto, y también el subempleo visible e invisible. La tasa de desempleo aumentó situándose en un 6.2\% lo que significa aproximadamente 76.000 personas desocupadas, 12.000 más que el año anterior. La tasa de subutilización de la fuerza de trabajo alcanzó casi el $14 \%$. Por su parte el número absoluto de hogares pobres que no satisfacieron sus necesidades alimentarias llegó a 93.331. Ello indica que la recesión económica y la transición hacia una economía más abierta y menos regulada, tienen efectos excluyentes para importantes sectores de la población" (Op. Cit.; 1997, p. 33).

En el mismo documento se anota que persiste la política de reducción de ocupados del sector público, y que los directores, gerentes y administradores, fueron el grupo ocupacional en que más evidenció la reducción relativa del empleo.

También las personas con menor instrucción son las que han sido desplazadas de sus trabajos, pero sospechamos que, paralelamente el movimiento que se ejerce es de sustitución por mano de obra nicaragüense indocumentada, quienes llegan a ocupar aquellos puestos poco atractivos para los y las costarricenses, y para las instancias empleadoras significa ventajoso, en tanto compra su fuerza de trabajo por un precio $60 \%$ 
más bajo que si fuera una persona costarricense. Estos puestos se ubican en actividades como: cogidas de café, zafra, corta de banano, cosecha de cítricos y melón, así como en la construcción y oficios domésticos (Op. Cit, 1997).

El fenómeno que sobrevive a estos datos y que hemos tratado de exteriorizar, es que se está configurando el mercardo laboral y la vida cotidiana, con una diversificación inédita, en consecuencia, pareciera no existir una capacidad endógena para generar nuevas tecnologías y nuevos empleos calificados. Si mantenemos la grosera metáfora de campo de aterrizaje, nos veremos con la situación descrita al inicio, es decir pendulando entre la inclusión y la exclusión, situación que provee de empleos para los cuales no es indispensable una escolaridad avanzada que van a ser ocupados por los más cualificados, y con base en el "efecto dominó" otros van a ser desplazados, acentuándose de esta manera el subempleo, (el subempleo visible aumentó de $3.7 \%$ en 1995 a $4.4 \%$ en 1996).

También es cierto que estos empleos coexistirán con otros que están en la esfera de la microelectrónica (importada), que requieren otras capacidades y conocimientos donde se encontrará la mayor parte de la fuerza de trabajo, por último un pequeño grupo ubicado en la gestión propiamente dicha, es decir profesionales especializados.

Esta situación no es perenne, por el contrario es totalmente temporal y móvil y por qué no incierta; dado que como se ha notado, nuestra estructura productiva está fuertemente influída por el ritmo en el desarrollo del conocimiento producido en los países u organismos centrales el cual es cada vez más rápido, donde constantemente se presentan nuevos avances, se resignifican las nociones de tiempo y espacio, que obligan a reconfigurar el campo laboral local. Este movimiento por lo tanto exige repensar la configuración del sistema educativo.

Consideramos que el panorama no es para nada halagüeño, hoy más que nunca la acción del Estado en el tema de redistribución del gasto social, se torna relevante. Debemos crear estrategias para asegurarnos que el gasto social tenga un impacto progresivo, como 
contener la reproducción de situaciones extremas como las que expresan los datos de 1996 donde el gasto en educación per cápita fue de 29,545 colones. También los procedimientos de ingreso en los diferentes niveles e instituciones plantean formas poco democráticas y susceptibles de ser revisadas. Es probable que cada vez más personas en edad escolar se retiren de la escuela para "incorporarse" en el mercado de trabajo. Esto es precisamente lo que los Organismos Multilaterales destacan para proponer la financiación de la educación básica, en tanto supone que habría una mayor tasa de retorno social, por cuanto es a la que están accediendo la mayor cantidad de personas y de todos los grupos sociales.

Con lo anterior queremos decir que la distribución del financiamiento por nivel no puede ni debe ser una acción mecánica o aislada como problema exclusivo de la educación. Si consideramos que el conocimiento es necesario para la existencia misma de las personas, la educación es fundamental y debe abarcar todos los niveles existentes en la actualidad, no se puede prescindir de ninguno. Pero pensamos que debe ser el conocimiento de la compleja situación social y cultural la que nos debe llevar a tomar decisiones en ese sentido, para lograr la equidad e igualdad que pregonamos y que no se garantizan solamente con una adecuada distribución de los recursos.

\section{3. ¿Afilar machetes para qué?}

Los sistemas educativos -dicen los expertos en el documento "Objetivos, criterios y lineamientos” de la CEPAL-UNESCO“- están experimentando una suerte de rigidez institucional con acciones lentas que lo conforman impenetrable a las demandas y desafíos externos, producto de su centralización, burocratización y encapsulamiento corporativo.

El deterioro de la calidad educativa es puesto en agenda y se verifica que la relación de años de estudio y el acceso a los conocimientos socialmente significativos no es tan real. La eficiencia como parte de este macro problema, generalmente ha sido evaluada al menos, desde dos indicadores: repetición y desgranamiento, (Filmus; 1995). 
Pero es lógico pensar que el desgranamiento y repetición no se produce independientemente de la situación socio-económica de las familias y las comunidades a las cuales pertenecen los y las estudiantes. En este sentido recordemos que en 1996, 45.146 hogares no lograron satisfacer sus necesidades alimentarias y que 96331 hogares no satisfacieron sus necesidades básicas no alimentarias, es decir, que un $21,6 \%$ son hogares pobres y $6,9 \%$ viven en extrema pobreza y el $14,7 \%$ no satisfacen las necesidades básicas, y que gran parte de esta población constituyen las comunidades como Los Guido, Los Cuadros o como Río Azul, con una alta propensión a la delincuencia, que viven de lo que extraen del basurero municipal y que a su alrededor se ha instalado un mercado "negro" de productos deshechados, vencidos o reciclados,.

A pesar de que durante los últimos años, el Ministerio de Educación se ha empeñado por elevar la eficiencia de la educación, los datos reflejan que se ha caminado lentamente en ese aspecto. El desgranamiento en el nivel primario, ha pasado de $38,1 \%$ en 1990 a 35,6\% en 1996, para los mismos años, en el nivel de III Ciclo, los porcentajes se incrementan de 59,3\% a 62,6 \%. En menor medida, el nivel de Educación Diversificada corre la misma suerte, al pasar de $73,5 \%$ a $73,7 \%$ de desgranamiento.

La repetición de primer grado durante los años 1992, 1994 y 1996 son respectivamente $18.635 ; 18.320$ y 19,938 personas, es notable "el incremento de la deserción escolar durante los últimos años; en 1996 fue del 4,5\% en el I y II ciclos y en secundaria, tres veces más que en primaria, 13,8\%”.(Estadode la Nación, 1997:63).

El desgranamiento (la repetición y la deserción), interpretado a la luz de la situación de pobreza, desempleo y subempleo, en que viven una buena parte de los hogares costarricenses, hace pensar que el problema trasciende lo educativo institucional por lo que creemos que los motivos que influyen para que las y los estudiantes permanezcan en las aulas, están cruzados por factores relacionados con la sobrevivencia. Pero esto a su vez 
genera nuevos problemas en tanto que la práctica experimentada es que a menor instrucción menos posibilidades de permanecer incluídos en el mundo del trabajo.

Las cifras de personas que se incorporan como mano de obra en edades tempranas, reflejan que al menos el $1,8 \%$ de la fuerza de trabajo tienen edades entre 12 a 14 años y el 10\% tienen edades entre 15 a 19 años, por otro lado 145268 personas, es decir el $17 \%$ de la población en edad escolar, -de 12 a 19 años- se integraron en actividades generadoras de ingresos, el $78 \%$ de ellos tiene un nivel de instrucción de primaria incompleta, sólo el $25 \%$ de la población de trece años o más ha completado al menos diez años de estudio. Por último, de los alumnos que ingresaron a $7^{\circ}$ año en 1986, únicamente una tercera parte logró graduarse (E.L.N.; 1995 y 1997).

Sería un eufemismo tratar de analizar la eficiencia de la educación como un problema propio e independiente. Los datos expuestos aun cuando corresponden a un número de rubros limitados, plantean lo complejo que resulta querer dar respuesta a la "ineficiencia" del Sistema Educativo. En otras palabras si los dos indicadores que presentamos como evaluadores de la calidad de la educación, se analizan independientemente de sus respectivos contextos, presentarán relaciones erróneas y poco coherentes con la realidad.

Esto pareciera ha sido lo que ha permitido al Banco Mundial proponer fórmulas para solventar el dilema de la baja calidad como la de transferir las escuelas a los gobiernos municipales. El Estado, establecería los contenidos mínimos comunes y el marco legal, mientras las municipalidades o estados provinciales incluirían contenidos particulares dependiendo de las realidades específicas.

El financiamiento será proporcionado por el sector público municipal, los recursos fiscales son transferidos con base al número de alumnos matriculados y el acceso será gratuito. Con esto se reemplazaría el subsidio actual a la oferta educacional por un apoyo por alumno es decir subsidiando la demanda. 
Este modelo de financiamiento, puede ser aplicable en los Estado-nación que han satisfecho las necesidades básicas de la sociedad, de esa manera las municipalidades podrían disponer de recursos para financiar la educación con diferencias mínimas entre ellas, con lo que se obtendría un nivel de calidad no tan heterogéneo como sucede en los países pobres.

De esta manera, en un proyecto de descentralizar la eficiencia de la educación ${ }^{5}$ se encontrará sometido a las contingencias de las relaciones de poder político y adquisitivo de las municipalidades, con tendencia a la profundización de las condiciones asimétricas y promoción de las desigualdades y las diferencias tanto de la calidad como de las condiciones de vida de los actores involucrados en el proceso (docentes, estudiantes, familias, etc) Esto puede redundar en el incremento de las migraciones puesto que los que tienen recursos optarán por mejores opciones; en el clientelismo político, en tanto que la priorización de asistencia no se encuentra exento de los intereses partidarios, entre otros factores.

Creemos que sin una política estatal adecuada para redistribuir equitativamente los recursos materiales y simbólicos, que aseguren la simetría entre las provincias, no es posible pensar en la eficiencia de la descentralización del sistema educativo. Aunado a esto es indispensable propiciar un cambio en los patrones culturales para que el proceso sea asumido responsablemente por parte de todos los actores intervinientes. Insistimos que lo contrario nos lleva a la construcción de "feudos" donde unos estarán más próximos a participar de la vida nacional que otros que lucharán por no "caerse" y quedar excluídos.

\section{El delicado oficio de afilar machetes}

La educación puede asumirse como un bien mixto que comparte características de bien privado y de bien público, lo primero comprendido como generador de beneficios 0

\footnotetext{
${ }^{5}$ Se trata de transferir la responsabilidad tanto técnica como económica a las provincias para que sean ésas, municipalidades mediante las que administren la estructura educativa
} 
utilidad interna divisible, apropiable solamente por el individuo y el segundo como el que genera beneficios o utilidad externa indivisible, apropiable por toda la comunidad. Así los conocimientos, habilidades o aptitudes adquiridos por una persona le proporcionan un diferencial de ingreso que lo disfruta solo ella, como individual es el disfrute el hecho de estudiar. Pero simultáneamente asisten otros beneficios indirectos de los cuales se apropia la comunidad; cuanto más educada sea una comunidad, hay mejores condiciones de vida. En síntesis hablamos de mejores tasas de retorno individual y social (Pifano; s.f.).

Esta consideración denota la importancia social que tiene la educación y nos conduce a repensar su finalidad. Tal como se ha expuesto pareciera que el Sistema Educativo, ya no se condice con la realidad que nos está tocando vivir (por lo que se corre el riesgo de estar formando para la frustración en un caso, en otro desde la educación privada para la permanencia incluída).

El desempleo, la pobreza y todas las consecuencias asociadas a ella, la descualificación, el desgranamiento, la deserción entre otros factores, son parte de esa constelación de elementos que deben atravesar una propuesta de reforma educativa. La alternativa de sistema educativo, debe fundarse en la formación integral del ser humano, y el desarrollo justo, solidario, participativo e igualitario de la sociedad, esto es un compromiso urgente.

Esta urgencia no es posible sin la presencia protagónica del Estado en los procesos educativos, para tratar de repensar la acción que debe ejercer éste en la construcción de una plataforma indisoluble de equidad e igualdad para el acceso a la educación; en otras palabras, se debe garantizar el reconocimiento tanto de la diferencia como de la desigualdad de los demandantes para gestionar el logro de la universalización del ingreso con una oferta redistributiva de recursos materiales y simbólicos de igual calidad.

Desde luego, esto nos obliga estar vigilantes para no someter a la educación a la lógica del mercado, lo que puede resultar complejo y peligroso presentándose una tendencia 
a la reificación, privando además la sugerencia de parcializar la problemática y considerarla como exclusivo de lo ofrecido institucionalmente, dejando de lado el sentido continuo que ella tiene.

Asimismo se debe refrendar el contrato real de que el Estado no financie la demanda sino, que lo haga a partir de la oferta. Esto porque propiciar la igualdad de oportunidades mediante el financiamiento de la demanda, significa promover una educación selectiva, y profundizar la segmentación social e incrementar exclusiones, es sustentar que al ingreso de la escuela, las necesidades básicas están satisfechas. Esto hace pensar en la urgencia que tiene el Estado, para despejar qué clase de ser humano ciudadano quiere formar, replanteando a su vez la relación sociedad, educación y mundo del trabajo. Luego, las estrategias de financiamiento se asumirán en la articulación de la equidad e igualdad.

Hay que repensar la educación y la inserción que debe tener en este nuevo orden, que viene a nuestro encuentro y que simultáneamente nos arrastra a él, y preguntarse si es viable mantener la distribución del conocimiento por disciplinas, por niveles, segmentado en materias, ordenado en planes de estudio rígidos, que luego desembocan en certificados, si pronto nos veremos ejerciendo oficios y profesiones y realizando tareas en plural. Cómo debemos organizar el conocimiento para que las personas, se apropien de él, y dispongan de criticidad, capacidades y competencias adecuadas al movimiento tecnológico y puedan asegurarse el aterrizaje de capitales para no caer en la exclusión.

Dada la inestabilidad que provoca este proceso, donde no se tienen puestos fijos ni la seguridad de permanecer incluido, donde la automatización produce nuevas formas de organización laboral, son necesarios nuevos contenidos curriculares que le provean a la fuerza de trabajo una actitud crítica ante lo que se avecina, una sólida formación general, capacidad de pensamiento teórico abstracto, sólida formación lógico-matemático, estadística e informática que acceda a una formación polivalente, polifuncional y flexible. 
Esta criticidad abrirá posibilidades para otras competencias relacionadas con la toma de decisiones, capacitación permanente, comunicación, planificación, entre otros, que serán necesararias, para evitar que se acrecienten las exclusiones (Filmus, 1994).

La idea que nos surge es una institución que oferte las capacidades y competencias en un continnun, organizada en una suerte de módulos híbridos, flexibles, en donde las personas entren y salgan en correspondencia con las nuevas necesidades sociales y demandas laborales que se les presente, esto porque la recualificación asegura la diversidad de opciones para acceder al mundo del trabajo y en el mejor de los casos la relativa permanencia.

Insistimos en que el Estado debe ser el actor protagónico en esta propuesta. Acentuar la privatización de la Educación significa acrecentar las desigualdades y la exclusión. Desde esta perspectiva la descentralización en condiciones óptimas podría ser una manera de reorganizar el sistema educativo, como instancia de educación permanente y continua, coherente con las necesidades sociales y demandas del mercado, en procura de la formación ciudadana. 


\section{REFERENCIAS}

Azpiazu, D.; E. Basualdo; H. Notcheff. (1988). La revolución tecnológica y las políticas hegemónicas. El complejo electrónico en la Argentina. Buenos Aires: Lesaga.

Banco Mundial. (1986). El financiamiento de la educación en los países en desarrollo. Opciones de política. Washington, Estados Unidos.

CEPAL-UNESCO. (1992). Objetivos, criterios y lineamientos. Santiago, Chile: UNESCO

CEPAL-UNESCO. (1992). Educación y conocimiento como eje de la transformación productiva con equidad. Santiago, Chile: UNESCO

Coraggio, J.L. (1992). Economía y Educación en América Latina: Notas para una agenda de los 90. Santiago de Chile: Consejo de Educación de Adultos de América Latina.

Estado de la Nación. (1997). Estado de la nación en desarrollo humano sostenible. San José, Costa Rica: Programa Estado de La Nación.

Estado, sociedad y educación en la Argentina de fin de siglo. Proceso y desafíos. (1996). Buenos Aires: Troquel Educación.

Filmus, D. (1994). El papel de la educación frente a los desafíos de las transformaciones científico-tecnológicas. En: Para qué sirve la escuela. Buenos Aires: Grupo Editorial Norma.

Fundación de Investigaciones Económicas Latinoamericanas (FIEL).

(1993). Descentralización de la escuela primaria y media. Una propuesta de reforma. Buenos Aires: FIEL.

La educación es un paracaídas. (1996, 28 de marzo). En: Clarín. Buenos Aires.

Labarca, G. (1981). Economía Política de la educación. México: Ediciones Nueva Imagen.

Paiva, V. (1992). Educación, bienestar social y trabajo. Buenos Aires: Libros del Quirquincho.

Piffano, H. (1996). Tópicos sobre la economía de la Educación. Buenos Aires: Material mimeografiado.

Tedesco, J.C. (1995). El nuevo pacto educativo. Educación, competitividad y ciudadanía en la sociedad moderna. Madrid: Grupo Amaya. 